\title{
Cholesterol level regulates lectin-like oxidized low-density lipoprotein receptor-1 function
}

\author{
Sofia Raniolo, Giulia Vindigni and Silvia Biocca* \\ Department of Systems Medicine, University of Rome Tor Vergata, Via Montpellier 1, 00133, Rome, \\ Italy
}

\begin{abstract}
Lectin-like oxidized low-density lipoprotein receptor-1 (LOX-1), the primary receptor for ox-LDL in endothelial cells, is a multi-ligand scavenger receptor that plays a crucial role in the pathogenesis of atherosclerosis and cardiovascular disorders and recently identified as a tumor marker. LOX-1 is naturally present in caveolae/lipid rafts in plasma membranes and disruption of these membrane domains by cholesterol-lowering drugs leads to a spatial disorganization of LOX-1 and a marked loss of specific LOX-1 function in terms of ox-LDL binding and internalization. Moreover, cholesterol depletion triggers the release of LOX-1 in exosomes and enhances shedding of LOX-1 ectodomain.

We here provide an overview of the involvement of membrane and circulating cholesterol in LOX-1 function and shedding and its impact on cardiovascular pathologies and cancer. In particular, we consider the available biological and molecular evidence indicating LOX-1 as a potential therapeutic target for atherosclerosis, inflammation processes, myocardial infarction and cholesterol-lowering drugs as specific inhibitors of LOX-1 function.
\end{abstract}

Keywords: Cholesterol, LOX-1 receptor, ox-LDL, lipid rafts, $\mathrm{M} \beta \mathrm{CD}$, statins

\section{Abbreviations}

CTLD C-type lectin-like domain

DiI 1, 1' -dioctadecyl-3,3,3',3'-tetramethyllindocarbocyanine perchlorate

ECs endothelial cells

HMG-CoA 3-hydroxy-3-methylglutaryl coenzyme A

LDL low-density lipoprotein

LOX-1 lectin-like oxidized low-density lipoprotein receptor-1

$\mathrm{M} \beta \mathrm{CD} \quad$ methyl- $\beta$-cyclodextrin

ox-LDL oxidized low-density lipoprotein.

\footnotetext{
${ }^{*}$ Corresponding author. Tel.: +39 72596418; Fax: +39 72596407; E-mail: biocca@med.uniroma2.it.
} 


\section{Introduction}

Cholesterol is the major sterol present in animal tissues and is an essential component of cell membranes. It is involved in many cellular processes, such as membrane trafficking, endocytosis, signal transduction, myelin formation, ions transport, synaptogenesis and steroid hormones biosynthesis. It regulates the biological properties of cell membranes since it reduces membrane fluidity and permeability by compacting phospholipids. It also defines the functional properties of membrane-resident proteins such as scavenger, transmitter and growth factor receptors [13,56].

Normal cells obtain cholesterol from two sources: endogenous synthesis and receptor-mediated uptake of circulating low-density lipoprotein (LDL). These particles contain about 1600 molecules of cholesteryl esters and 170 triglyceride molecules, forming a central lipophilic core surrounded by an outer monolayer, comprised of about 700 phospholipid molecules (mainly phosphatidylcholine and sphingomyelin), 600 molecules of unesterified cholesterol, a variable amount of antioxidants and 1 molecule of ApoB-100 protein [32]. The internalized LDL is endocyted and traffics to lysosomes where the cholesteryl esters are hydrolyzed, and the cholesterol is released for new membrane synthesis. At low cholesterol levels, cells activate the gene for HMG CoA reductase, the rate-controlling enzyme in the cholesterol biosynthetic pathway, as well as the genes for LDL receptors thereby allowing cells to synthesize cholesterol [23]. When cellular cholesterol levels rise, transcription of the LDL receptor gene decreases. This feedback system keeps the level of cholesterol in cell membranes constant [11].

Over the years, cholesterol has gained a bad reputation in the world of health and nutrition, especially because of its association with cardiovascular diseases. Elevated cholesterol levels were associated with an increase in the incidence of heart attacks, and the uptake of LDL by macrophage with the consequent foam cell formation was identified as the link between elevated levels of serum LDL and heart disease. When circulating LDL increases to high levels in plasma for a prolonged time, it eventually settles in arteries creating atherosclerotic plaques [11]. Under pathological condition, LDL deposits in vascular tissue are rapidly converted to modified-LDL by reactive molecular species generated by the endothelium, smooth muscle tissue and migratory lymphocytes. The oxidation of LDL is a complex process, which involves changes to both protein and non protein moieties $[48,64]$. During oxidation, free radicals react with the polyunsaturated fatty acids (mostly arachidonic acid and linoleic acid) present in the outer core of lipoprotein particles, while ApoB-100 undergoes to an extensive fragmentation due to non-enzymatic oxidative cleavage, mainly on histidine, lysine and proline residues [1,20]. The major consequence of oxidation is the increase of negative charges on LDL surface, which is crucial for receptor recognition. After oxidation, LDL becomes unrecognizable by the LDL receptor, but binds to several scavenger receptors (SR), including SR-A I/II, CD36, SR-BI, FcgRII and lectin-like oxidized low-density lipoprotein receptor-1 (LOX-1) [35].

LOX-1 was originally identified as the main receptor of ox-LDL in endothelial cells [62]. Under physiological conditions, basal cellular LOX-1 expression is low in most tissues, while it is up-regulated in several pathophysiological processes such as inflammation, atherosclerosis, obesity and diabetes $[59,68]$. In this context, LOX-1 and ox-LDL contribute to plaque rupture in atherosclerosis by promoting lipid accumulation, oxidative stress, pro-inflammatory response, release of metalloproteinases and apoptotic cell death.

In this review, we consider the involvement of cholesterol in normal LOX-1 receptor function and its impact on cardiovascular pathologies and concentrate on recent findings indicating LOX-1 as a potential therapeutic target for atherosclerosis, inflammation processes, myocardial infarction and cancer. Moreover, we describe the cell model system, which allows the overexpression of the human LOX-1 
receptor mimicking the pathological conditions, and the fluorescent-based assay used for studying the ox-LDL-LOX-1 receptor binding and internalization.

\section{LOX-1 receptor}

LOX-1 receptor belongs to the C-type lectin-like receptors family. It is a type II membrane glycoprotein composed by 273 residues and four domains: a short $\mathrm{N}$-terminal cytoplasmic domain (CYTO, 34 aa), a single transmembrane domain (TM, 26 aa), an extracellular region consisting of a coiledcoil domain named NECK (82 aa), and a C-type lectin-like domain (CTLD, 131 aa) at the C-terminus (Fig. 1) $[53,54,62]$. The CTLD domain forms a disulfide-linked heart-shaped homodimer, which assembles in larger functional oligomers through non-covalent interactions $[6,53,54]$. The crystal structure of the human LOX-1 receptor revealed the presence of arginine residues, forming a basic spine located on the CTLD and crossing the entire dimer, which are engaged in the ligand binding [53,54]. Molecular dynamics (MD) simulations evidenced that mutations of the basic spine residues Trp150 and Lys167 markedly reduces LOX-1 binding activity $[5,18,52,53]$. A hydrophobic tunnel runs through the center of the CTLD (Fig. 1(B)). The tunnel access is surrounded by a quasi-conical surface where hydrophilic

A

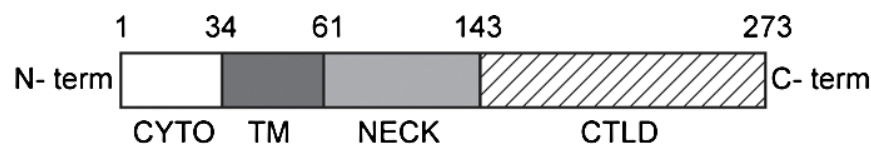

B

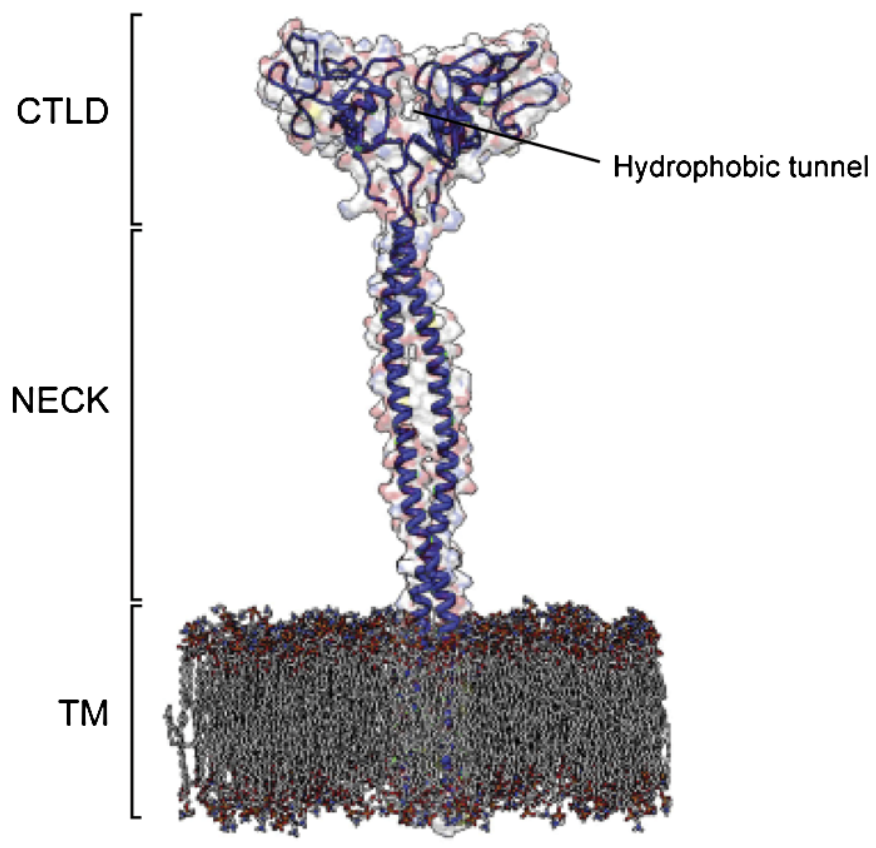

Fig. 1. (A) Schematic representation of the primary sequence of LOX-1. (B) LOX-1 three D model without cytoplasmic domain (reprinted with permission from reference [4]). The protein is represented by a blue ribbon inside a transparent surface, while membrane is depicted using stick model phospholipids. 
and hydrophobic patches are scattered. Mutations of residues that obstruct the tunnel and the presence of phospholipids that can interact with residues in the tunnel, significantly prevent the binding ability of LOX-1 receptor $[19,21]$. The NECK domain appears as a dimer consisting of two $\alpha$-helices wound in a parallel coiled-coil structure and displays specific residues, which functionally modulate the flexibility of this region [30].

LOX-1 is encoded by a single gene, orl1, mapped on human chromosome 12p12.3-p13.2 [2]. Genetic association studies have identified various polymorphisms (SNPs, single nucleotide polymorphisms) in orll gene [40], which are associated to an increased susceptibility to acute myocardial infarction. These SNPs modulate the expression of a splicing isoform of LOX-1 receptor, named LOXIN, which protects macrophages against ox-LDL-mediated apoptosis [40]. LOXIN is deficient in ox-LDL binding activity but interacts with LOX-1 receptors inhibiting its function through the formation of non-functional hetero-oligomers [6].

LOX-1 receptor recognises a wide range of negatively charged substances. In addition to ox-LDL, these ligands include activated platelets, apoptotic bodies, bacteria, advanced glycation end products (AGEs), heat shock proteins (HSP60 and HSP70) and C-reactive protein (CRP) [62,68,70]. Beside endothelial cells, LOX-1 is expressed in several cell types including smooth muscle cells, fibroblasts and platelets $[16,46,69]$. Under physiological conditions, it is expressed at low level and it plays a versatile role in innate immunity or infections and may be involved in the removal of cellular debris and aged/apoptotic cells from blood circulation. LOX-1 expression is induced by ligand binding via intracellular signaling in multiple pathological states, such as atherosclerosis, obesity, inflammation and in cellular transformation and carcinogenesis $[14,26]$. Its activation triggers the oxidative stress response causing plaque vulnerability and potential rupture, which leads to acute atherothrombotic vascular occlusion and tissue infarction [37,59]. The higher local concentration of ox-LDL, together with the higher expression of LOX-1 receptor, in atherosclerotic lesions, provides a molecular basis linking ox-LDL to endothelial cells and the resultant cellular activation, dysfunction, and injury. In animal models, deletion of orll gene in Ldlr knockout mice results in much smaller atherosclerosis lesions, drastic reduction of inflammation in aortic wall and of the extent of ischemia/reperfusion injury [28,45]. LOX-1 is also expressed on the surface of immune cells such as denditric cells and macrophages, where it contributes to inflammatory responses and foam cells formation, respectively [51]. Ox-LDL binding to LOX-1 receptor induces the activation of membrane-bound NADPH oxidase leading to a rapid increase of reactive oxygen species (ROS) and the consequent induction of the redox-sensitive NF-kB pathway $[59,68]$. Among the effects of the activation of NF-kB is the enhancement of pro-inflammatory cytokine and chemokine expression, such as MCP-1, VCAM-1, ICAM-1, E-selectin and P-selectin, with an increase of monocytes recruitment to endothelial cells [68].

More recently, different studies have shown a correlation between increased serum ox-LDL levels and risk of cancer. Several lipid metabolic genes, including orll, are consistently overexpressed in diverse cancer cell lineages and, importantly, the expression of these genes is critical to cellular transformation, as well as in maintaining the transformed state. A meta-analysis of gene expression profiles of 950 cancer cell lines stored in the Gene Expression Atlas at the EMBL-EBI database (http://www.ebi.ac.uk/gxa/ gene/ENSG00000173391\#) reveals that the LOX-1 gene is up-regulated in 57\% of bladder and cervix cancer cells, $11 \%$ of mammary gland cancer cells, $10 \%$ of lung cancer cells, and in $20 \%$ of colon rectal cancer (CRC) cells $[24,26,47]$. The expression of LOX-1 is modulated since the early stage of tumor development, suggesting a potential role in the insurgence and progression of the disease and associated with more aggressive and metastatic stage tumors. As in atherosclerosis, the ox-LDL-LOX-1 interaction 
activates the inflammatory pathway through NF- $\kappa \mathrm{B}$ activation [39] increases the expression of proatherogenic molecules such as VEGF, MMP-2 and MMP-9 and, in cancer cells stimulates proliferation [24]. Depletion of LOX-1 receptors protects against tumorigenicity, motility and growth of these cells $[26,47]$.

Altogether, these findings suggest that therapies for the inhibition of LOX-1 receptors may be effective in reducing the rate of atherosclerotic, inflammation processes and cancer progression.

\section{A cell-based assay for measuring LOX-1 function}

Receptor oligomerization is a common mechanism by which cell surface receptors increase the avidity of ligand binding. This is true, for example, for the TNF family of receptors, G-protein coupled and receptors for advanced glycation end products (RAGE) $[8,55,67]$. Multimerization and cluster organization in plasma membranes are important requisites also for LOX-1 high affinity binding of ligands, as demonstrated in vitro and in vivo [5,6,12,42,52-54]. In fact, comparing the size of LOX-1 CTLD $(\approx 70 \AA)$ with the ox-LDL diameter $(\approx 250 \AA)$, it seems realistic that the LOX-1 binds to ox-LDL as an assembly of clustered receptors.

To investigate the ligand binding function of cell surface LOX-1 receptors in cells, we have set up a cell-based assay. The human recombinant LOX-1 was transiently transfected in COS fibroblasts and found to be correctly expressed and localized [6]. The LOX-1-ox-LDL binding in this cell system can be monitored by labeling ox-LDL with the highly fluorescent lipophilic dye 1,1'-diocadecyl-3-3-3', $3^{\prime}$ tetramethyl indocarbocyanine perchlorate dye (DiI), that diffuses into the hydrophobic portion of the LDL complex without affecting the LDL-specific binding of the apoprotein. Figure 2(A) shows a representative image of DiI-ox-LDL (red fluorescence) binding $\left(1 \mathrm{~h}\right.$ at $\left.4^{\circ} \mathrm{C}\right)$ to LOX-1 expressing COS cells. Since COS cells do not express endogenous LOX-1, only transfected cells specifically bind labelled oxLDL. The binding specificity is confirmed by incubating with 100-fold excess of cold ox-LDL, which completely abolishes DiI-ox-LDL binding to LOX-1 (Fig. 2(B)). This cell system allows a high transfection efficiency (about 40-50\% of transfected cells) and a very strong expression level of LOX-1 receptors on cell membrane, mimicking the in vivo characteristics of pathological states. Taking advantage of this assay, the efficacy of potential inhibitors and treatments to displace the binding of fluorescent DiI-ox-LDL to LOX-1 can be measured: (i) by counting positive DiI-ox-LDL fluorescent cells and/or

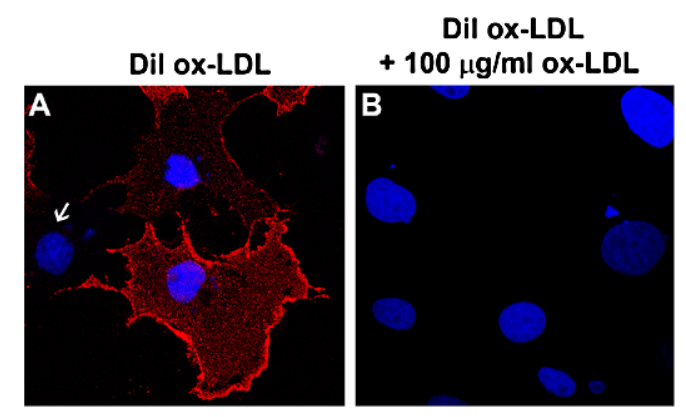

Fig. 2. Cell-based assay for monitoring LOX-1-ox-LDL interaction. (A) Cells were incubated with $10 \mu \mathrm{g} / \mathrm{ml}$ of DiI-ox-LDL at $4^{\circ} \mathrm{C}$ for $60 \mathrm{~min}$. Typical membrane fluorescence of DiI-ox-LDL was detectable only in LOX-1 transfected cells. Nuclei are blue stained with Hoechst 33342. White arrow indicates one not-transfected cell. (B) Specific binding of ox-LDL was measured as displaceable binding in the presence of $100 \mu \mathrm{g} / \mathrm{ml}$ of unlabeled ox-LDL. 
(ii) by extracting bound DiI-ox-LDL from positive stained cells with isopropanol and spectrofluorometric analysis [7]. The assay allows quantifying the inhibitory effect, being $100 \%$ of binding the amount of fluorescence in not-treated cells. Most of the experimental studies on the effect of cholesterol level on LOX-1 activity described below have been performed with this cell-based system.

\section{LOX-1 localization and membrane cholesterol}

The role of cholesterol in cellular function has gained interest after the proposal of the lipid raft hypothesis [43,63]. Lipid rafts are small $(200 \mathrm{~nm})$ heterogeneous, highly dynamic, cholesterol- and sphingolipid-enriched domains that compartmentalize cellular processes. In biological membranes, mixtures of saturated and unsaturated lipids separate into macroscopic domains of a liquid-ordered (lipid rafts) and a liquid-disordered phase. The raft phase is enriched in both cholesterol and saturated lipids; the non-raft phase consists mainly of the unsaturated lipids and is poor in cholesterol [61]. Initially proposed to function in protein sorting events in polarized cells, lipid rafts function as platforms that concentrate and segregate proteins within the plane of the bilayer [57] and regulate membrane trafficking in both the exocytic and endocytic pathways, cell migration, and a variety of cell signaling cascades [10]. To the formation of lipid rafts, lipid-lipid interactions are important, with cholesterol playing a special role on keeping these domains together [3]. Specific membrane proteins preferentially associate with one type of domain or the other. The presence of lipid rafts enhances protein-protein interactions for proteins contained within the same raft while inhibiting others by segregating raft- and non-raft proteins.

We have investigated the requirement of cholesterol for LOX-1 surface distribution and function and found that LOX-1 is mainly localized in caveolae/lipid rafts in the plasma membrane [42]. This finding was based on several well-established raft analysis techniques. First, LOX-1 receptors co-localize with filipin, a fluorescent marker of membrane cholesterol. Second, cholesterol sequestration by methyl- $\beta$ cyclodextrin ( $\mathrm{M} \beta \mathrm{CD}$ ), which specifically extracts cholesterol from the plasma membranes [29], induces the mislocalization of LOX-1 in membranes. LOX-1 molecules result to be in a more diffuse distribution without a reduction of the amount of receptors exposed on the cell surface, as depicted in Fig. 3.

By fractionation of cellular membranes and purification of cholesterol-rich lipid rafts we were able to show that functional LOX-1 is found almost entirely in a fraction containing caveolin-1, a marker of the caveolae/lipid raft fraction, in all LOX-1 expressing cell types including human primary endothelial cells [42].

\section{Statins regulate LOX-1 expression and function}

Statins are a family of cholesterol-lowering drugs used to lower total and LDL cholesterol. They were initially isolated from a penicillium mold as inhibitors of 3-hydroxy-3-methyl-glutaryl coenzyme A (HMG-CoA) reductase, the first enzyme in the cholesterol biosynthetic pathway [17]. Nowadays, statins are the principal therapeutic agents for atherosclerotic cardiovascular diseases and dyslipidemias. The protective effects of statins include prevention of endothelial dysfunction, enhanced plaque stability, anti-inflammatory effects, and correction of prothrombotic tendencies.

Notably, a long term exposure with different statins (lovastatin, atorvastatin, fluvastatin and pravastatin) results in a chronic inhibition of cholesterol biosynthesis and leads to a marked reduction of LOX-1 in lipid rafts and consequent reduction of ox-LDL binding and uptake [42]. In Fig. 4(A) histograms show quantification of DiI-ox-LDL binding measuring fluorescence by spectrofluorometer of cells treated with 
A

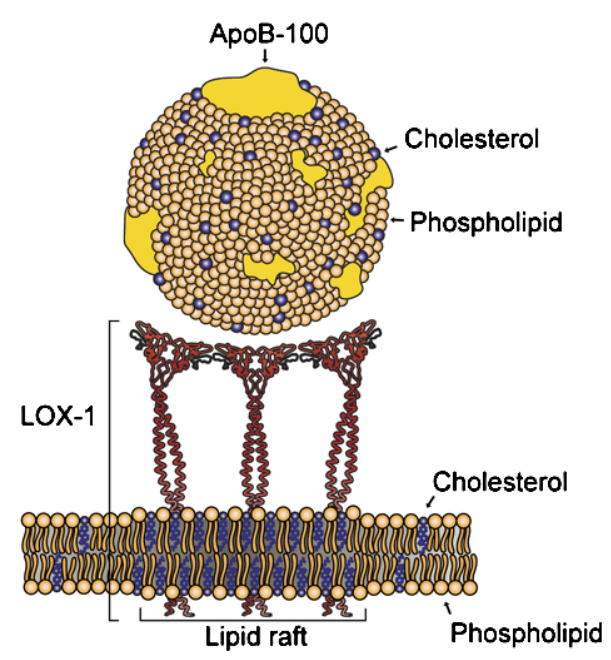

B
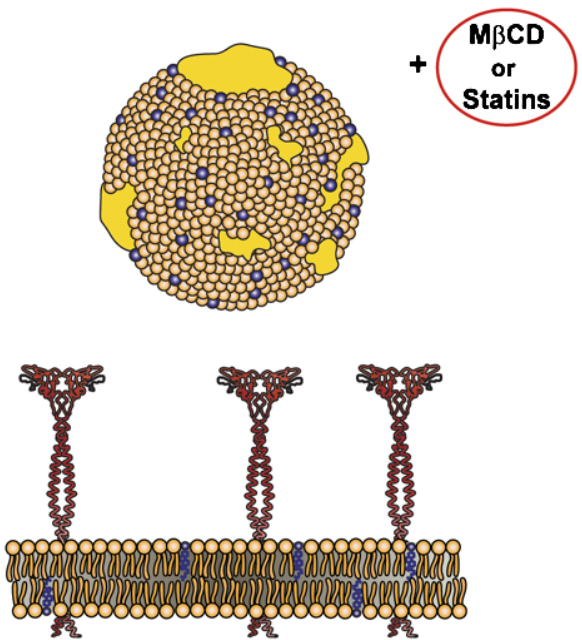

Cholesterol-lowering drugs effect

Fig. 3. Schematic drawing of the binding between three LOX-1 molecules and one ox-LDL particle in membranes with normal cholesterol content (A) and in cholesterol-depleted membranes (B).

A

\section{Long-term statin exposure}

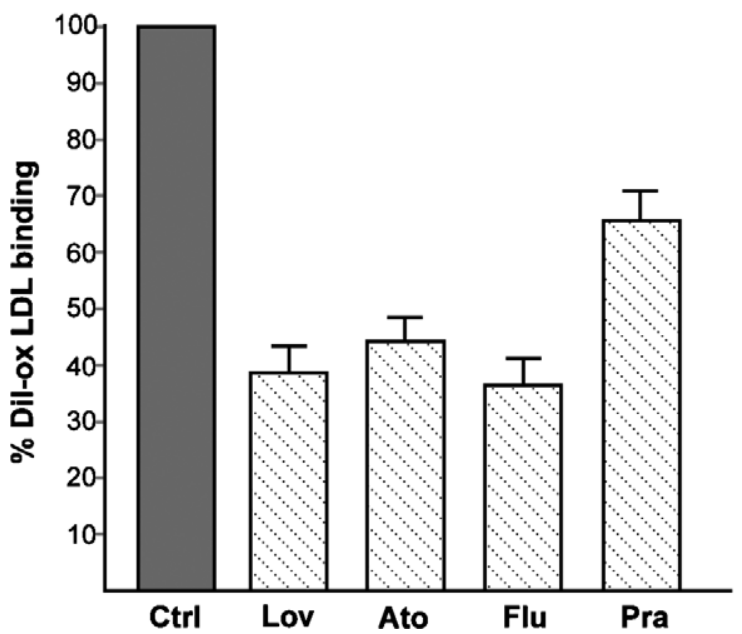

B

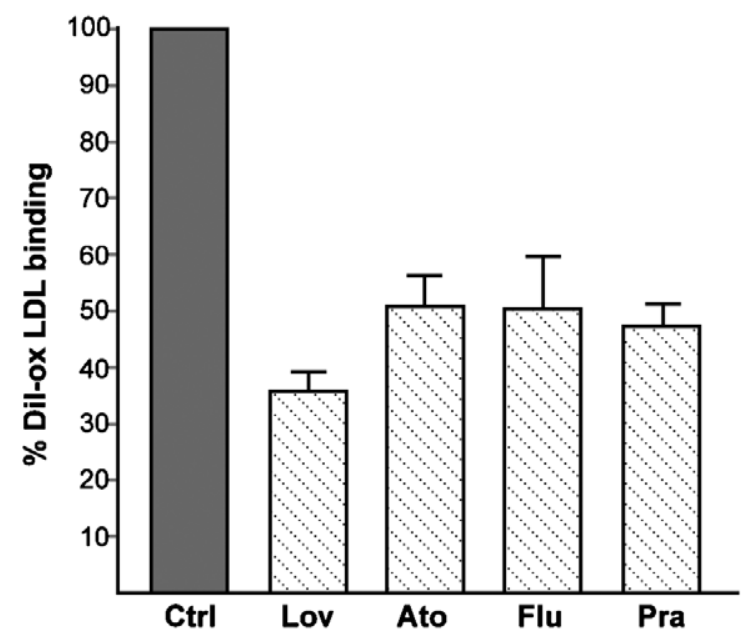

Fig. 4. (A) Effect of long-term exposure of statins on LOX-1-mediated ox-LDL binding. COS cells transiently transfected with human recombinant LOX-1 were treated or not (Ctrl) with $2 \mu \mathrm{M}$ of different statins (as indicated) for 24 hours. (B) Effect of short exposure of statins on LOX-1-mediated ox-LDL binding. COS cells transiently transfected with human recombinant LOX-1 were incubated with $10 \mu \mathrm{g}$ DiI-ox-LDL at $4^{\circ} \mathrm{C}$ for $60 \mathrm{~min}$ in the absence $(\mathrm{Ctrl})$ or in the presence of $2 \mu \mathrm{M}$ of different statins as indicated. Histograms show quantification of DiI-ox-LDL binding measuring fluorescence by spectrofluorometer. The data represent the mean \pm standard deviation calculated from 4 separate experiments. 
$2 \mu \mathrm{M}$ lovastatin (Lov), atorvastatin (Ato), fluvastatin (Flu) and pravastatin (Pra) for 24 hours. Inhibition of the intensity of the signal of DiI-ox-LDL bound to LOX-1 compared to the control value (Ctrl) is very strong, reaching $64 \%$ reduction of LOX-1 binding after treatment with fluvastatin. This new effect of statins on LOX-1 inhibition indicates that statins protect vascular endothelium against the adverse effect of ox-LDL by loss of LOX-1 receptor function [42]. Although several studies have already reported the effects of statins on LOX-1 expression in animal and cellular models, none of these reports has focused on the relationship between the potent cholesterol lowering effect of statins and LOX-1 function. In particular, statins induce a decrease in LOX-1 expression and ox-LDL uptake in human coronary artery endothelial cells [36,38], monocytes and macrophages [16,27], aortic SMCs [27], and platelets [41,60]. In endothelial cells, inhibition of LOX-1 by statins is associated with multiple anti-atherosclerotic effects, such as reduced uptake of ox-LDL, decreased apoptosis of endothelial cells, reduced monocyteEC adhesion, up-regulation of eNOS, and inhibition of Ang-II-converting enzyme (ACE) expression $[33,36,38,44]$. Administration of rosuvastatin reduces the up-regulation of LOX-1, associated NADPH oxidase and NF- $\kappa$ B activation in $\mathrm{LDLR}^{-1-}$ mice [33]. Simvastatin was found to suppress LOX-1 expression, ERK activation and to induce inhibition of proliferation of vascular smooth muscle cells in response to mechanical stretching with or without ox-LDL [71].

Although decrease of circulating or membrane-associated cholesterol is considered the principal beneficial effect of statins, these drugs also have cholesterol-independent responses. Thus, inhibition of the early step in cholesterol biosynthesis leads in turn to the inhibition of the synthesis of isoprenoid intermediates, which act as lipid anchors required for membrane tethering and activation of several proteins such as heterotrimeric G proteins and small GTP-binding proteins (in particular the family of Ras, Rho, Rap and Rab GTPases) [66].

We have further investigated the statin-mediated LOX-1 inhibition mechanism and found a new pleiotropic effect of statins, i.e. a direct interaction with LOX-1. All tested statins (lovastatin, atorvastatin, fluvastatin and pravastatin) are able to displace the binding of fluorescent ox-LDL to LOX-1 inhibiting LOX-1 activity [7]. In detail, short exposure with different statins at $4^{\circ} \mathrm{C}$ and for a time length not sufficient to induce any cholesterol synthesis reduction $(60 \mathrm{~min})$, results in a very marked decrease of ox-LDL bound to LOX-1 (Fig. 4(B)). Incubation with $2 \mu \mathrm{M}$ lovastatin induces a reduction of DiI-oxLDL bound to LOX-1 of $62 \%$ compared to the control value (100\%), while atorvastatin, fluvastatin and pravastatin reduce the fluorescence signal of $45 \%, 46 \%$ and $41 \%$ respectively. The ability of LOX-1 to adapt to various molecules is expected, being LOX-1 a scavenger receptor, involved in the removal of many foreign substances and waste materials by extensive ligand specificity.

Molecular docking simulations indicate that statins completely fill the hydrophobic tunnel that crosses the C-type lectin-like (CTLD) recognition domain of LOX-1, blocking the binding to natural substrates, including ox-LDL [7]. As mentioned above the hydrophobic tunnel plays a crucial role in the recognition of ox-LDL and other ligands. Single aminoacid mutations on Ile149, which points to the empty space in the center of the tunnel, leads to a marked inhibition of the binding of ox-LDL and a series of oxidized phospholipids [19,21]. Similarly to these compounds, our experimental and simulative investigation confirms that statins completely fill the hydrophobic tunnel using a common mechanism and that residue Ile149 is always involved in stabilizing contacts with these drugs [7].

\section{Cholesterol level modulates LOX-1 shedding}

Previous studies have shown that high levels of ox-LDL up-regulate LOX-1 receptor expression at the cell surface and enhance the proteolytic ectodomain shedding of a soluble form of LOX-1 (sLOX-1) [49, 
58]. This molecule has been identified in conditioned media and can be measured in serum and plasma. Although the significance of the soluble form of the receptor in circulating blood and the mechanisms of sLOX-1 release remain unclear, sLOX-1 appears to be a biomarker for acute coronary syndrome [25], for rheumatoid arthritis [31], for rupture of thin-cap fibroatheroma [34], for preeclampsia [65] and an early predictor of the metabolic syndrome [15]. A cleavage site within the NECK domain between Arg88 and Gln89 has been experimentally detected [4].

We have recently demonstrated that membrane cholesterol affects the release of different forms of LOX-1 in cells transiently and stably expressing human LOX-1 and in human permanent vein umbilical endothelial cells (EA.hy926) [22]. In detail, cholesterol depletion triggers the release of (i) LOX-1 in exosomes as a full-length transmembrane isoform and (ii) sLOX-1, the truncated ectodomain soluble fragment. Reduction of membrane cholesterol content and disruption of lipid rafts and caveolae facilitate sLOX-1 shedding. It was found that LOX-1 shedding is a metalloprotease-dependent process, possibly performed by a soluble matrix metalloproteinase, although the identification of the specific enzyme is still under study [22]. The previous observation that cholesterol promotes LOX-1 clustering in lipid rafts [42] envisages the possibility that clustering of LOX-1 in lipid rafts protects LOX-1 from proteolytic attack.

\section{Conclusions}

LOX-1, the main receptor for ox-LDL in endothelial cells, plays a crucial role in atherogenesis and inflammation processes. High circulating cholesterol level leads to production of ox-LDL particles, the natural substrate of LOX-1 that in turn induce up-regulation of LOX-1 receptors. High cholesterol also leads to the increase of membrane-associated cholesterol, stabilizing the raft localization of functional LOX-1 receptors. Both the decrease of cholesterol and the inhibition of LOX-1 function may be effective in reducing the rate of atherosclerotic and inflammation processes. Specific LOX-1 receptors inhibitors are not yet available and urgently necessary.

Accumulating evidence highlights a new beneficial effect of cholesterol-lowering drugs (statins) on blocking LOX-1 function with two distinct mechanisms: (i) by disrupting cholesterol-enriched regions of cell membranes, affecting LOX-1 localization and function and (ii) by a direct interaction of statins with the CTLD recognition domain of LOX-1. It is worth noting that a number of clinical investigation strongly support that statins have a marked beneficial effect in preventing myocardial damage after coronary angioplasty $[9,50]$. The description of these new pleiotropic effects of statins on LOX-1 function provides the basis for further studies opening a new field of investigation about possible different uses of these drugs and therapeutic intervention.

\section{References}

[1] P.M. Abuja and H. Esterbauer, Simulation of lipid peroxidation in low-density lipoprotein by a basic "skeleton" of reactions, Chem Res Toxicol. 8 (1995), 753-763. doi:10.1021/tx00047a016.

[2] T. Aoyama, T. Sawamura, Y. Furutani, R. Matsuoka, M.C. Yoshida, H. Fujiwara and T. Masaki, Structure and chromosomal assignment of the human lectin-like oxidized low-density-lipoprotein receptor-1 (LOX-1) gene, Biochem J. 339 (1999), 177-184. doi:10.1042/bj3390177.

[3] Y. Barenholz, Cholesterol and other membrane active sterols: From membrane evolution to "rafts", Prog Lipid Res. 41 (2002), 1-5. doi:10.1016/S0163-7827(01)00016-9. 
[4] S. Biocca, T. Arcangeli, E. Tagliaferri, B. Testa, G. Vindigni, F. Oteri, A. Giorgi, F. Iacovelli, G. Novelli, A. Desideri and M. Falconi, Simulative and experimental investigation on the cleavage site that generates the soluble human LOX-1, Arch Biochem Biophys 540 (2013), 9-18. doi:10.1016/j.abb.2013.10.001.

[5] S. Biocca, M. Falconi, I. Filesi, F. Baldini, L. Vecchione, R. Mango, F. Romeo, G. Federici, A. Desideri and G. Novelli, Functional analysis and molecular dynamics simulation of LOX-1 K167N polymorphism reveal alteration of receptor activity, PLoS One 4 (2009), e4648. doi:10.1371/journal.pone.0004648.

[6] S. Biocca, I. Filesi, R. Mango, L. Maggiore, F. Baldini, L. Vecchione, A. Viola, G. Citro, G. Federici, F. Romeo and G. Novelli, The splice variant LOXIN inhibits LOX-1 receptor function through hetero-oligomerization, Journal of Molecular and Cellular Cardiology 44 (2008), 561-570. doi:10.1016/j.yjmcc.2007.11.017.

[7] S. Biocca, F. Iacovelli, S. Matarazzo, G. Vindigni, F. Oteri, A. Desideri and M. Falconi, Molecular mechanism of statinmediated LOX-1 inhibition, Cell Cycle 14 (2015), 1583-1595. doi:10.1080/15384101.2015.1026486.

[8] J.L. Bodmer, P. Schneider and J. Tschopp, The molecular architecture of the TNF superfamily, Trends Biochem Sci 27 (2002), 19-26. doi:10.1016/S0968-0004(01)01995-8.

[9] C. Briguori, G. Visconti, A. Focaccio, B. Golia, A. Chieffo, A. Castelli, M. Mussardo, M. Montorfano, B. Ricciardelli and A. Colombo, Novel approaches for preventing or limiting events (Naples) II trial: Impact of a single high loading dose of atorvastatin on periprocedural myocardial infarction, J Am Coll Cardiol 54 (2009), 2157-2163. doi:10.1016/j.jacc.2009.07.005.

[10] D.A. Brown and E. London, Functions of lipid rafts in biological membranes, Annu Rev Cell Dev Biol. 14 (1998), 111136. doi:10.1146/annurev.cellbio.14.1.111.

[11] M.S. Brown and J.L. Goldstein, A receptor-mediated pathway for cholesterol homeostasis, Science 232 (1986), 34-47. doi: $10.1126 /$ science. 3513311.

[12] W. Cao, V. Calabro, A. Root, G. Yan, K. Lam, S. Olland, J. Sanford, A. Robak, R. Zollner, Z. Lu, M. Ait-Zahra, R. Agostinelli, L. Tchistiakova, D. Gill, D. Harnish, J. Paulsen and H.H. Shih, Oligomerization is required for the activity of recombinant soluble LOX-1, FEBS J 276 (2009), 4909-4920. doi:10.1111/j.1742-4658.2009.07190.x.

[13] N.M.F.S.A. Cerqueira, E.F. Oliveira, D.S. Gesto, D. Santos-Martins, C. Moreira, H.N. Moorthy, M.J. Ramos and P.A. Fernandes, Cholesterol biosynthesis: A mechanistic overview, Biochemistry 55 (2016), 5483-5506. doi:10.1021/ acs.biochem.6b00342.

[14] M. Chen, T. Masaki and T. Sawamura, LOX-1, the receptor for oxidized low-density lipoprotein identified from endothelial cells: Implications in endothelial dysfunction and atherosclerosis, Pharmacol Ther. 95 (2002), 89-100. doi:10.1016/ S0163-7258(02)00236-X.

[15] S. Civelek, M. Kutnu, H. Uzun, F. Erdenen, E. Altunoglu, G. Andican, A. Seven, A.O. Sahin and G. Burcak, Soluble lectin-like oxidized LDL receptor 1 as a possible mediator of endothelial dysfunction in patients with metabolic syndrome, J Clin Lab Anal (2014). doi:10.1002/jcla.21748.

[16] G. Draude, N. Hrboticky and R.L. Lorenz, The expression of the lectin-like oxidized low-density lipoprotein receptor (LOX-1) on human vascular smooth muscle cells and monocytes and its down-regulation by lovastatin, Biochemical Pharmacology 57 (1999), 383-386. doi:10.1016/S0006-2952(98)00313-X.

[17] A. Endo, M. Kuroda and Y. Tsujita, ML-236A, ML-236B, and ML-236C, new inhibitors of cholesterogenesis produced by Penicillium citrinum, J. Antibiot 29 (1976), 1346-1348. doi:10.7164/antibiotics.29.1346.

[18] M. Falconi, S. Biocca, G. Novelli and A. Desideri, Molecular dynamics simulation of human LOX-1 provides an explanation for the lack of OxLDL binding to the Trp150Ala mutant, BMC Struct Biol 7 (2007). doi:10.1186/1472-6807-7-73.

[19] M. Falconi, S. Ciccone, P. D’Arrigo, F. Viani, R. Sorge, G. Novelli, P. Patrizi, A. Desideri and S. Biocca, Design of a novel LOX-1 receptor antagonist mimicking the natural substrate, Biochem Biophys Res Commun 438 (2013), 340-345. doi:10.1016/j.bbrc.2013.07.073.

[20] L.G. Fong, S. Parthasarathy, J.L. Witztum and D. Steinberg, Nonenzymatic oxidative cleavage of peptide bonds in apoprotein B-100, J Lipid Res. 28 (1987), 1466-1477.

[21] O.L. Francone, M. Tu, L.J. Royer, J. Zhu, K. Stevens, J.J. Oleynek, Z. Lin, L. Shelley, T. Sand, Y. Luo and C.D. Kane, The hydrophobic tunnel present in LOX-1 is essential for oxidized LDL recognition and binding, J Lipid Res $\mathbf{5 0}$ (2009), 546-555. doi:10.1194/jlr.M800474-JLR200.

[22] M. Gioia, G. Vindigni, B. Testa, S. Raniolo, G.F. Fasciglione, M. Coletta and S. Biocca, Membrane cholesterol modulates LOX-1 shedding in endothelial cells, PLoS One 10 (2015), e0141270. doi:10.1371/journal.pone.0141270.

[23] J.L. Goldstein and M.S. Brown, A century of cholesterol and coronaries: From plaques to genes to statins, Cell 161 (2015), 161-172. doi:10.1016/j.cell.2015.01.036.

[24] I. González-Chavarría, R.P. Cerro, N.P. Parra, F.A. Sandoval, F.A. Zuñiga, V.A. Omazábal, L.I. Lamperti, S.P. Jiménez, E.A. Fernandez, N.A. Gutiérrez, F.S. Rodriguez, S.A. Onate, O. Sánchez, J.C. Vera and J.R. Toledo, Lectin-like oxidized LDL receptor-1 is an enhancer of tumor angiogenesis in human prostate cancer cells, PLoS One 9 (2014), e106219. doi:10.1371/journal.pone.0106219.

[25] K. Hayashida, N. Kume, T. Murase, M. Minami, D. Nakagawa, T. Inada, M. Tanaka, A. Ueda, G. Kominami, H. Kambara, T. Kimura and T. Kita, Serum soluble lectin-like oxidized low-density lipoprotein receptor-1 levels are ele- 
vated in acute coronary syndrome: A novel marker for early diagnosis, Circulation 112 (2005), 812-818. doi:10.1161/ CIRCULATIONAHA.104.468397.

[26] H.A. Hirsch, D. Iliopoulos, A. Joshi, Y. Zhang, S.A. Jaeger, M. Bulyk, P.N. Tsichlis, X.S. Liu and K.A. Struhl, Transcriptional signature and common gene networks link cancer with lipid metabolism and diverse human diseases, Cancer Cell 13 (2010), 348-361. doi:10.1016/j.ccr.2010.01.022.

[27] O. Hofnagel, B. Luechtenborg, H. Eschert, G. Weissen-Plenz, N.J. Severs and H. Robenek, Pravastatin inhibits expression of lectin-like oxidized low-density lipoprotein receptor-1 (LOX-1) in Watanabe heritable hyperlipidemic rabbits: A new pleiotropic effect of statins, Arterioscler Thromb Vasc Biol. 26 (2006), 604-610. doi:10.1161/01.ATV. 0000201073.45862.8b.

[28] C. Hu, J. Chen, A. Dandapat, Y. Fujita, N. Inoue, Y. Kawase, K. Jishage, H. Suzuki, D. Li, P.L. Hermonat, T. Sawamura and J.L. Metha, LOX-1 abrogation reduces myocardial ischemia-reperfusion injury in mice, J Mol Cell Cardiol 44 (2008), 76-83. doi:10.1016/j.yjmec.2007.10.009.

[29] S. Ilangumaran and D.C. Hoessli, Effects of cholesterol depletion by cyclodextrin on the sphingolipid microdomains of the plasma membrane, Biochem J. 335 (1998), 433-440. doi:10.1042/bj3350433.

[30] T. Ishigaki, I. Ohki, N. Utsunomiya-Tate and S. Tate, Chimeric structural stabilities in the coiled-coil structure of the NECK domain in human lectin-like oxidized low-density lipoprotein receptor 1 (LOX-1), J Biochem 14 (2007), 855-866. doi:10.1093/jb/mvm093.

[31] M. Ishikawa, H. Ito, M. Akiyoshi, N. Kume, H. Yoshitomi, H. Mitsuok, S. Tanida, K. Murata, H. Shibuya, T. Kasahara, A. Kakino, Y. Fujita, T. Sawamura, T. Yasuda and T. Nakamura, Lectin-like oxidized low-density lipoprotein receptor 1 signal is a potent biomarker and therapeutic target for human rheumatoid arthritis, Arthritis \& Rheumatism 64 (2012), 1024-1034. doi:10.1002/art.33452.

[32] W. Jessup, L. Kritharides and R. Stocker, Lipid oxidation in atherogenesis: An overview, Biochem Soc Trans 32 (2004), 134-138. doi:10.1042/bst0320134.

[33] B.Y. Kang and J.L. Mehta, Rosuvastatin attenuates Ang II-mediated cardiomyocyte hypertrophy via inhibition of LOX-1, J Cardiovasc Pharmacol Ther. 14 (2009), 283-291. doi:10.1177/1074248409344329.

[34] N. Kobayashi, N. Hata, N. Kume, S. Yokoyama, M. Takano, T. Shinada, K. Tomita, A. Shirakabe, T. Inami, Y. Seino and K. Mizuno, Detection of acute aortic dissection by extremely high soluble lectin-like oxidized LDL receptor-1 (sLOX-1) and low troponin T levels in blood, Int J Cardiol 165 (2013), 557-559. doi:10.1016/j.ijcard.2012.09.001.

[35] I. Levitan, S. Volkov and P.V. Subbaiah, Oxidized LDL: Diversity, patterns of recognition, and pathophysiology, Antioxidants and Redox Signaling 13 (2010), 39-75. doi:10.1089/ars.2009.2733.

[36] D. Li, H. Chen, F. Romeo, T. Sawamura, T. Saldeen and J.L. Mehta, Statins modulate oxidized low-density lipoproteinmediated adhesion molecule expression in human coronary artery endothelial cells: Role of LOX-1, J Pharmacol Exp Ther 302 (2002), 601-605. doi:10.1124/jpet.102.034959.

[37] D. Li, V. Williams, L. Liu, H. Chen, T. Sawamura, F. Romeo and J.L. Mehta, Expression of lectin-like oxidized lowdensity lipoprotein receptors during ischemia-reperfusion and its role in determination of apoptosis and left ventricular dysfunction, J Am Coll Cardiol. 41 (2003), 1048-1055. doi:10.1016/S0735-1097(02)02966-2.

[38] D.Y. Li, H.J. Chen and J.L. Mehta, Statins inhibit oxidized-LDL-mediated LOX-1 expression, uptake of oxidized-LDL and reduction in PKB phosphorylation, Cardiovasc Res 52 (2001), 130-135. doi:10.1016/S0008-6363(01)00371-6.

[39] J. Lu, S. Mitra, X. Wang, M. Khaidakov and J.L. Mehta, Oxidative stress and lectin like ox-LDL receptor LOX-1 in atherogenesis and tumorigenesis, Antioxid Redox Signal. 15 (2011), 2301-2333. doi:10.1089/ars.2010.3792.

[40] R. Mango, S. Biocca, F. del Vecchio, F. Clementi, F. Sangiuolo, F. Amati, A. Filareto, S. Grelli, P. Spitaleri, I. Filesi, C. Favalli, R. Lauro, J.L. Metha, F. Romeo and G. Novelli, In vivo and in vitro studies support that a new splicing isoform of OLR1 gene is protective against acute myocardial infarction, Circulation Research 97 (2005), 152-158. doi:10.1161/01. RES.0000174563.62625.8e.

[41] M.R. Marwali, C.P. Hu, B. Mohandas, A. Dandapat, P. Deonikar, J. Chen, I. Cawic, T. Sawamura, M. Kavdia and J.L. Mehta, Modulation of ADP-induced platelet activation by aspirin and pravastatin: Role of lectin-like oxidized lowdensity lipoprotein receptor-1, nitric oxide, oxidative stress, and inside-out integrin signaling, J Pharmacol Exp Ther 322 (2007), 1324-1332. doi:10.1124/jpet.107.122853.

[42] S. Matarazzo, M.C. Quitadamo, R. Mango, S. Ciccone, G. Novelli and S. Biocca, Cholesterol-lowering drugs inhibit lectin-like oxidized low-density lipoprotein-1 receptor function by membrane raft disruption, Molecular Pharmacology 82 (2012), 246-254. doi:10.1124/mol.112.078915.

[43] F.R. Maxfield and I. Tabas, Role of cholesterol and lipid organization in disease, Nature 438 (2005), 612-621. doi:10.1038/ nature04399.

[44] J.L. Mehta, D.Y. Li, H.J. Chen, J. Joseph and F. Romeo, Inhibition of LOX-1 by statins may relate to upregulation of eNOS, Biochem Biophys Res Commun. 289 (2001), 857-861. doi:10.1006/bbrc.2001.6070.

[45] J.L. Mehta, N. Sanada, C.P. Hu, J. Chen, A. Dandapat, F. Sugawara, H. Satoh, K. Inoue, Y. Kawase, K. Jishage, H. Suzuki, M. Takeya, L. Schnackenberg, R. Beger, P.L. Hermonat, M. Thomas and T. Sawamura, Deletion of LOX-1 reduces athero- 
genesis in LDLR knockout mice fed high cholesterol diet, Circ Res 100 (2007), 1634-1642. doi:10.1161/CIRCRESAHA. 107.149724.

[46] H. Moriwaki, N. Kume, T. Sawamura, T. Aoyama, H. Hoshikawa, H. Ochi, E. Nishi, T. Masaki and T. Kita, Ligand specificity of LOX-1, a novel endothelial receptor for oxidized low density lipoprotein, Arterioscler Thromb Vasc Biol. 18 (1998), 1541-1547. doi:10.1161/01.ATV.18.10.1541.

[47] M. Murdocca, R. Mango, S. Pucci, S. Biocca, B. Testa, R. Capuano, R. Paolesse, M. Sanchez, A. Orlandi, C. di Natale, G. Novelli and F. Sangiuolo, The lectin-like oxidized LDL receptor-1: A new potential molecular target in colorectal cancer, Oncotarget 7 (2016), 14765-14780. doi:10.18632/oncotarget.7430.

[48] M. Navab, G.M. Ananthramaiah, S.T. Reddy, B.J. Van Lenten, B.J. Ansell, G.C. Fonarow, K. Vahabzadeh, S. Hama, G. Hough, N. Kamranpour, J.A. Berliner, A.J. Lusis and A.M. Fogelman, The oxidation hypothesis of atherogenesis: the role of oxidized phospholipids and HDL, J Lipid Res. 45 (2004), 993-1007. doi:10.1194/jlr.R400001-JLR200.

[49] T. Navarra, S. Del Turco, S. Berti and G. Basta, The lectin-like oxidixed low-density lipoprotein receptor 1 and its soluble form: Cardiovascular implications, Journal of Atherosclerosis and Thrombosis 17 (2010), 317-331. doi:10.5551/jat.3228.

[50] A. Nusca, R. Melfi, G. Patti and G. Di Sciascio, Statin loading for acute coronary syndromes, Curr Opin Cardiol 25 (2010), 373-378. doi:10.1097/HCO.0b013e32833987ca.

[51] S. Oh and H. Joo, LOX-1 boosts immunity, Oncotarget 6 (2015), 21763-21764. doi:10.18632/oncotarget.4756.

[52] I. Ohki, H. Amida, R. Yamada, M. Sugihara, T. Ishigaki and S. Tate, Surface plasmon resonance study on functional significance of clustered organization of lectin-like oxidized LDL receptor (LOX-1), Biochim Biophys Acta 1814 (2011), 345-354. doi:10.1016/j.bbapap.2010.10.006.

[53] I. Ohki, T. Ishigaki, T. Oyama, S. Matsunaga, Q. Xie, M. Ohnishi-Kameyama, T. Murata, D. Tsuchiya, S. Machida, K. Morikawa and S. Tate, Crystal structure of human lectin-like, oxidized low-density lipoprotein receptor 1 ligand binding domain and its ligand recognition mode to OxLDL, Structure 13 (2005), 905-917. doi:10.1016/j.str.2005.03.016.

[54] H. Park, F.G. Adsit and J.C. Boyington, The 1.4 angstrom crystal structure of the human oxidized low density lipoprotein receptor LOX-1, J Biol Chem 280 (2005), 13593-13599. doi:10.1074/jbc.M500768200.

[55] P.S. Park, S. Filipek, J.W. Wells and K. Palczewski, Oligomerization of G protein-coupled receptors: Past, present, and future, Biochemistry 43 (2004), 15643-15656. doi:10.1021/bi047907k.

[56] F.W. Pfrieger, Cholesterol homeostasis and function in neurons of the central nervous system, Cell Mol Life Sci. 60 (2003), 1158-1171. doi:10.1007/s00018-003-3018-7.

[57] L.J. Pike, Rafts defined: A report on the keystone symposium on lipid rafts and cell function, J Lipid Res. 47 (2006), $1597-1598$

[58] A. Pirillo and A.L. Catapano, Soluble lectin-like oxidized low density lipoprotein receptor 1 (sLOX-1) as a biochemical marker for atherosclerosis related diseases, Dis. Markers 35 (2013), 413-418. PMID: 24198442. doi:10.1155/ 2013/716325.

[59] A. Pirillo, G.D. Norata and A.L. Catapano, LOX-1, OxLDL, and atherosclerosis, Mediators Inflamm 2013 (2013), 152786. doi: $10.1155 / 2013 / 152786$.

[60] L. Puccetti, T. Sawamura, A.L. Pasqui, M. Pastorelli, A. Auteri and F. Bruni, Atorvastatin reduces platelet-oxidized-LDL receptor expression in hypercholesterolaemic patients, Eur J Clin Invest 35 (2005), 47-51. doi:10.1111/j.1365-2362. 2005.01446.x.

[61] H.J. Risselada and S.J. Marrink, The molecular face of lipid rafts in model membranes, Proc Natl Acad Sci USA 105 (2008), 17367-17372. doi:10.1073/pnas.0807527105.

[62] T. Sawamura, N. Kume, T. Aoyama, H. Moriwaki, H. Hoshikawa, Y. Aiba, T. Tanaka, S. Miwa, Y. Katsura, T. Kita and T. Masaki, An endothelial receptor for oxidized low density lipoprotein, Nature 386 (1997), 73-77. doi:10.1038/ $386073 \mathrm{a} 0$.

[63] K. Simons and E. Ikonen, Functional rafts in cell membranes, Nature 387 (1997), 569-572. doi:10.1038/42408.

[64] D. Steinberg, Low density lipoprotein oxidation and its pathobiological significance, J Biol Chem. 272 (1997), 2096320966. doi:10.1074/jbc.272.34.20963.

[65] A. Tuten, B. Aydemir, M. Oncul, A.R. Kiziler, A.S. Acıkgoz, G.G. Korkmaz, V. Sozer and H. Uzun, The association of lectin-like oxidized LDL receptor 1 (LOX-1) K167N and 3'UTR188CT polymorphisms with maternal plasma soluble LOX-1 levels and preeclampsia risk in Turkish population, Arch Gynecol Obstet 291 (2014), 563-571. doi:10.1007/ s00404-014-3457-4.

[66] C.Y. Wang, P.Y. Liu and J.K. Liao, Pleiotropic effects of statin therapy: Molecular mechanisms and clinical results, Trends Mol Med. 14 (2008), 37-44. doi:10.1016/j.molmed.2007.11.004.

[67] J. Xie, S. Reverdatto, A. Frolov, R. Hoffmann, D.S. Burz and A. Shekhtman, Structural basis for pattern recognition by the receptor for advanced glycation end products (RAGE), J Biol Chem 283 (2008), 27255-27269. doi:10.1074/jbc. M801622200.

[68] S. Xu, S. Ogura, J. Chen, P.J. Little, J. Moss and P. Liu, LOX-1 in atherosclerosis: Biological functions and pharmacological modifiers, Cell Mol Life Sci 70 (2013), 2859-2872. doi:10.1007/s00018-012-1194-z. 
[69] H. Yoshida, N. Kondratenko, S. Green, D. Steinberg and O. Quehenberger, Identification of the lectin-like receptor for oxidized low-density lipoprotein in human macrophages and its potential role as a scavenger receptor, Biochemical Journal 334 (1998), 9-13. doi:10.1042/bj3340009.

[70] R. Yoshimoto, Y. Fujita, A. Kakino, S. Iwamoto, T. Takaya and T. Sawamura, The discovery of LOX-1, its ligands and clinical significance, Cardiovasc Drugs Ther. 225 (2011), 379-391. doi:10.1007/s10557-011-6324-6.

[71] Z. Zhang, M. Zhang, Y. Li, S. Liu, S. Ping, J. Wang, F. Ning, F. Xie and C. Li, Simvastatin inhibits the additive activation of ERK1/2 and proliferation of rat vascular smooth muscle cells induced by combined mechanical stress and oxLDL through LOX-1 pathway, Cell Signal. 25 (2013), 332-340. doi:10.1016/j.cellsig.2012.10.006. 